\section{Infecciones parameníngeas: reporte de dos casos asociados con sinusitis}

\section{RESUMEN}

El absceso cerebral y el empiema subdural son infecciones parameníngeas graves y poco frecuentes en la actualidad. Se presentan un caso de absceso cerebral y uno de empiema subdural asociados con sinusitis; ambos fueron tratados con ceftriaxona, vancomicina y metronidazol (uno de ellos además con drenaje quirúrgico) y presentaron evolución clínica satisfactoria. Es importante la sospecha diagnóstica temprana para dar un tratamiento oportuno y mejorar el pronóstico en estos pacientes.

Palabras clave: absceso cerebral; empiema subdural.

\section{Parameningeal infections: report of 2 cases associated with sinusitis}

\begin{abstract}
Brain abscess and subdural empyema are serious and rare parameningeal infections. One case of brain abscess and another one of subdural empiema associated to sinusitis are presented. Both were treated with ceftriaxone, vancomycin and metronidazole (one of them also needed surgical drainage) and had satisfactory clinical outcome. An early diagnostic suspicion is important to provide timely treatment and thus improve prognosis in these patients.
\end{abstract}

Keywords: brain abscess; subdural empyema.

\author{
Napoleón González-Saldaña ${ }^{1}$ \\ Valeria Gómez-Toscano ${ }^{2}$ \\ ${ }^{1}$ Jefe del Departamento de Infectología. \\ ${ }^{2}$ Médico adscrito al Servicio de Infectología Pediá- \\ trica. Miembro titular de la Asociación Mexicana de \\ Infectología Pediátrica. \\ Instituto Nacional de Pediatría, México, D.F.
}

Recibido: 3 de noviembre del 2014

Aceptado: 28 de mayo del 2015

Correspondencia: Napoleón González Saldaña Jefe del Depto. de Infectología Instituto Nacional de Pediatría. Insurgentes Sur 3700- C CP 04530 México, D.F. Tel.: 10840009 ext. 1367 drnagosal@hotmail.com

Este artículo debe citarse como González-Saldaña N, Gómez-Toscano V. Infecciones parameníngeas: reporte de dos casos asociados con sinusitis. Acta Pediatr Mex 2016;37:32-37. 


\section{INTRODUCCIÓN}

Un absceso se puede localizar en el parénquima cerebral o en los espacios subdural o epidural. Puede originarse de infecciones contiguas (como osteomielitis de cráneo, sinusitis u otitis crónica), de anomalías vasculares subyacentes (cardiopatía congénita cianógena), después de traumatismo de cráneo o de procedimientos neuroquirúrgicos. Estas situaciones pueden alterar la función neurológica por destrucción directa del tejido nervioso, por compresión ocasionada por efecto de masa o por infarto secundario a obstrucción venosa o arterial.

\section{CASO CLÍNICO 1}

Niño 13 años de edad con antecedente de rinorrea y constipación nasal de larga evolución. Inició su padecimiento 13 días antes con tos; un día después tuvo fiebre de $38^{\circ} \mathrm{C}$, para lo cual recibió tratamiento sintomático. Al día siguiente hubo incremento de volumen a nivel del párpado superior izquierdo, aumento de la temperatura local, dolor y eritema, así como cefalea hemicraneana ipsilateral. Se le diagnosticó celulitis periorbitaria izquierda y se prescribió ceftriaxona intramuscular y tobramicina tópica. Hubo entonces incremento de la celulitis y continuó con cefalea intermitente de moderada intensidad. Se le llevó a un centro hospitalario donde se realizó drenaje de absceso palpebral; se obtuvieron $50 \mathrm{~mL}$ de material purulento.

Durante su internamiento aumentó la cefalea y la inflamación. Se trató con ceftriaxona y vancomicina por tres días y se refirió a este instituto con diagnóstico de celulitis orbitaria. El día de su traslado tuvo náusea y vómito en seis ocasiones.

A su ingreso, en la exploración física se le encontró somnoliento pero reactivo a estímulos externos. En ojo izquierdo se observó absceso palpebral de coloración violácea, incapacidad funcional, una herida de $2 \mathrm{~cm}$, sin proptosis, congestión nasal, cavidad oral con descarga verde y fétida. Neurológicamente sin rigidez de nuca ni datos de focalización; funciones mentales superiores conservadas.

La biometría hemática reportó hemoglobina $13.1 \mathrm{q} / \mathrm{dL}$; hematocrito $39.3 \%$; leucocitos $12,500 / \mu \mathrm{L}$; neutrófilos $81 \%$; linfocitos $17 \%$; plaquetas $601,000 / \mu \mathrm{L}$, proteína C reactiva $5.2 \mathrm{mg} /$ dL. Una tomografía axial de cráneo mostró un absceso cerebral frontal izquierdo (Figura 1).

Un día después se observó disminución de la escala de Glasgow a 9 puntos, anisocoria (pupila derecha de $2 \mathrm{~mm}$ e izquierda de $4 \mathrm{~mm}$ ), hipertensión y bradicardia. Se le realizó craniectomía bifrontal, cranealización del seno frontal, punción del absceso intracraneal, duroplastia con pericráneo y ventriculostomía precoronal derecha. Durante la cirugía se encontró edema cerebral acentuado, hernia transcalvaria de $3 \mathrm{~cm}$ y salida espontánea de líquido cefalorraquídeo purulento. No hubo crecimiento en cultivo. Recibió 42 días de tratamiento con ceftriaxona, vancomicina y metronidazol con evolución favorable $y$ fue egresado.

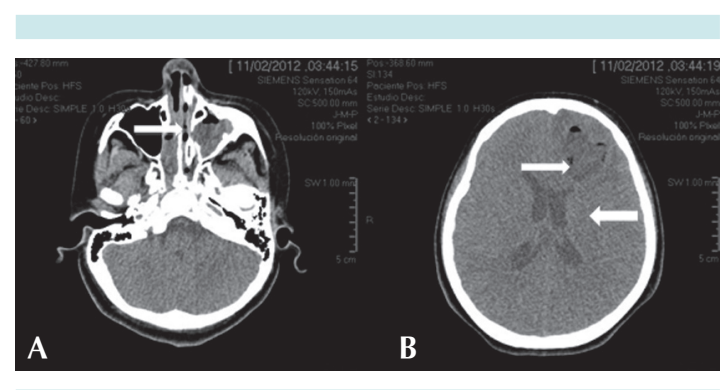

Figura 1. A) Sinusitis con extensión etmoidal (flecha). B) Lesión anular hipodensa en la sustancia blanca lobar frontal izquierda con diámetros de $3 \times 3 \mathrm{~cm}$ y aerocele menor a $1 \mathrm{~cm}^{3}$ sugerentes de absceso cerebral frontal izquierdo (flecha delgada). Edema perilesional y discreta compresión del asta frontal del ventrículo lateral izquierdo sin desviar estructuras de la línea media (flecha gruesa), sin comunicación a ventrículos ni al espacio subaracnoideo. 


\section{CASO CLÍNICO 2}

Niño de 14 años de edad. Tuvo sinusitis dos años antes del inicio de su padecimiento actual. Recibió tratamiento no especificado con el que alivió sus síntomas. Inició su padecimiento cuatro días antes a su ingreso con irritabilidad y cefalea pulsátil muy intensa (10/10) en la región frontal derecha. Se le diagnosticó migraña y recibió ketorolaco, diclofenaco, sumatriptán y betametasona sin presentar mejoría. Días después cursó con somnolencia, cambios en la conducta y vómito en proyectil de contenido gástrico. Se le diagnosticó cefalea tensional y continuó con analgésico. Un día antes de su ingreso, vómito 25 veces, lenguaje entrecortado, mirada perdida, dificultad para la marcha por disminución en la fuerza del hemicuerpo izquierdo, pérdida de la coordinación y fiebre no cuantificada. En otro hospital se corroboró hemiparesia izquierda e incapacidad para la marcha. Se le realizó una tomografía axial de cráneo en la que se observó ocupación de los senos frontales y etmoidales bilaterales e infarto cerebral en región parietal derecha. Fue referido a esta institución.

A su ingreso, el paciente se encontró alerta, orientado, con pupilas isocóricas y normorreflécticas, pares craneales conservados, movimientos oculares normales, reflejo nauseoso presente, pilares amigdalinos simétricos, reflejo cocleopalpebral, tono y trofismo normales, fuerza en hemicuerpo izquierdo $4 / 5$ y en hemicuerpo derecho $5 / 5$, reflejos de estiramiento muscular 3/4 de hemicuerpo izquierdo y $2 / 4$ de hemicuerpo derecho, respuesta plantar flexora, rigidez de nuca, signos de Kernig y Brudzinski positivos.

La biometría hemática reportó hemoglobina $15.6 \mathrm{q} / \mathrm{dL}$; hematocrito $45.6 \% \mathrm{M}$; leucocitos $35,300 / \mu \mathrm{L}$; neutrófilos $91 \%$; linfocitos $4 \%$; plaquetas $228,000 / \mu \mathrm{L} ; 5 \%$ bandas. La velocidad de sedimentación globular fue de $18 \mathrm{~mm} / \mathrm{h}$.
Se realizó punción lumbar y el citoquímico reportó aspecto turbio, proteínas $225 \mathrm{mg} / \mathrm{dL}$, glucosa $74 \mathrm{mg} / \mathrm{dL}$ (central $144 \mathrm{mg} / \mathrm{dL}$ ), células 11,808/ $\mathrm{mm}^{3}$, leucocitos $11,628 / \mathrm{mm}^{3}$, eritrocitos 180 / $\mathrm{mm}^{3}$, polimorfonucleares $89 \%$ y mononucleares $11 \%$. Gram, coaglutinación y cultivo fueron negativos. Se sospechó meningitis bacteriana y se indicó ceftriaxona, vancomicina y dexametasona.

Tres días después, presentó bradicardia, hipertensión y deterioro de la escala de Glasgow a 10 puntos. Requirió ventilación mecánica y terapia intensiva. Se realizó nueva tomografía de cráneo (Figura 2) que mostró empiema interhemisférico inoperable causado por pansinusitis complicada y edema cerebral secundario. Se agregó metronidazol al tratamiento.

La tomografía de control a los 8 días sugirió una adecuada respuesta al tratamiento antimicrobiano (Figura 3) por lo que se determinó que no requería drenaje intracraneal y sólo se realizó cirugía endoscópica de senos paranasales con drenaje de senos etmoidales. El informe de patología reportó sinusitis frontal etmoidal crónica quística bilateral sin eosinofilia. Egresó después de 38 días de tratamiento antibiótico con triple esquema. La exploración únicamente con hemiparesia izquierda que remitió posteriormente.

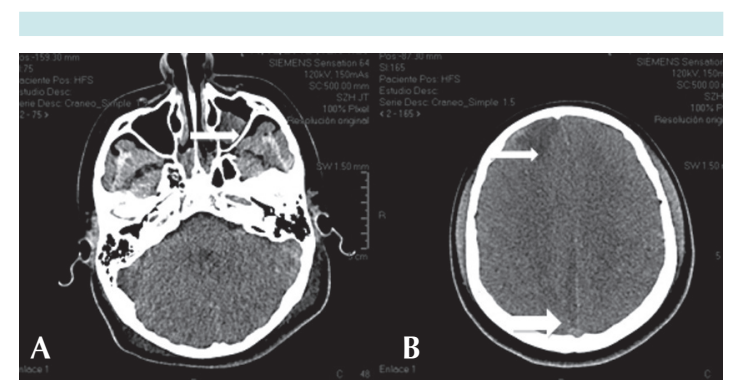

Figura 2. A) Pansinusitis (flecha). B) Imagen frontal derecha polar heterogénea sin reforzamiento en la periferia (flecha delgada) e imagen nueva hipodensa interhemisférica derecha homogénea de $0.4 \mathrm{~cm}$ de grosor, aunque sin efecto de volumen (flecha gruesa). 


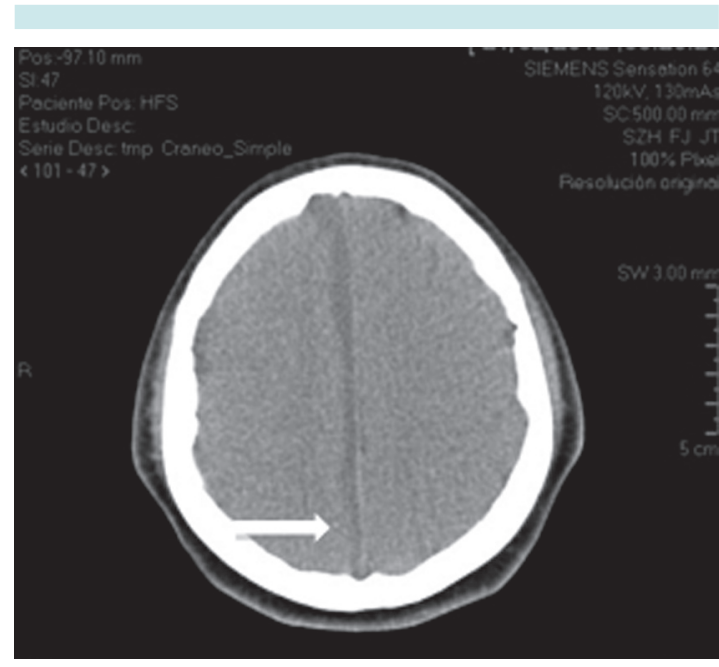

Figura 3. Mejoría en la colección interhemisférica, de $2 \mathrm{~mm}$ de grosor, con disminución de $2 \mathrm{~mm}$ comparados con la tomografía previa (flecha).

\section{DISCUSIÓN}

Las infecciones parameníngeas pueden deberse a diseminación de la infección por contigüidad a partir de un sitio extracraneal, a diseminación hematógena de un sitio de infección distante o a inoculación directa de microorganismos durante una lesión traumática penetrante..$^{1,2}$

La otitis y la mastoiditis pueden asociarse con formación de absceso a nivel temporal o cerebe$\operatorname{lar}^{3}$ y la sinusitis e infecciones dentales a nivel frontal, como en el primer caso. La diseminación metastásica de distintos focos en pacientes con cardiopatía congénita y con cortocircuito de derecha a izquierda condiciona afectación a cualquier nivel del parénquima, pero el origen más común proviene de los senos paranasales, del oído medio o de una infección dental. Existen entre 20 y 30\% de origen idiopático. ${ }^{1}$

Se han descrito cuatro estadios de un absceso cerebral: en el primero hay cerebritis (días 1 a 3), área focal de inflamación aguda, dilatación vascular, microtrombosis, ruptura de los vasos pequeños y edema. Luego, en el segundo estadio el centro de la lesión muestra licuefacción. En la fase de cerebritis tardía (días 4 a 9) se expande la cerebritis y se forma un centro necrótico. En el tercer estadio de cápsula temprana (días 10 a 14), como en el primer paciente al momento del ingreso, se caracteriza por la formación de una cápsula de colágeno densa de tejido vascularizado con gliosis periférica o fibrosis. Finalmente, durante el estado de cápsula tardía (más de 14 días) se observa la cápsula bien formada. ${ }^{1}$

Por otro lado, el empiema subdural es una afectación rara y puede encontrarse asociada con meningitis. En niños mayores la infección generalmente no sigue a una leptomeningitis, pues aunque una leptomeningitis puede originar el empiema subdural, usualmente una sinusitis u otomastoiditis crónica se disemina hacia el espacio subdural directamente debido a osteomielitis o a través de venas infectadas que penetran el cráneo, tal como ocurrió en el segundo caso. También puede ser de origen hematógeno. ${ }^{1}$

Los microorganismos predominantes en las infecciones parameníngeas son los estreptococos aerobios y anaerobios (60-70\% de los casos), bacilos anaerobios gramnegativos (20-40\%), enterobacterias (20-30\%), S. aureus (10-15\%) y hongos (1-5\%). Se aíslan múltiples microorganismos aerobios y anaerobios en un tercio de los pacientes. ${ }^{3}$ En los casos aquí reportados no se reportó aislamiento de ningún microorganismo, pero por el origen de la infección es de suponer que estuvieran involucrados, principalmente, estreptococos aerobios y anaerobios.

La presentación clínica, como en los casos previamente descritos, está dada por cefalea de inicio reciente asociada con uno o más de los siguientes síntomas: vómito, fotofobia, fiebre, crisis convulsivas y cambios en el estado de alerta (desde letargo hasta coma). Otros sínto- 
mas menos frecuentes son: disartria, debilidad o hemiparesia (estos datos presentes en el segundo caso), parestesias, disfagia, afasia y nistagmo. ${ }^{4}$ Cabe recalcar que ambos pacientes presentaron fiebre, cefalea, vómito y cambios en el estado de alerta; pero ninguno padeció crisis convulsivas.

El tratamiento puede ser médico o médico y quirúrgico. ${ }^{5}$ Está descrito sólo tratamiento médico en pacientes neurológicamente íntegros, sin datos de hipertensión intracraneal y en quienes el absceso se limita a la fase de cerebritis (padecimiento menor a 2 semanas), el absceso o abscesos son pequeños (menores de $3 \mathrm{~cm}$ de diámetro) o en pacientes muy inestables para someterse a procedimiento quirúrgico. ${ }^{1}$ El primer caso reunió los criterios para tratamiento quirúrgico. Por otro lado, aunque se ha descrito como indispensable el tratamiento quirúrgico en niños mayores con empiema subdural (craneotomía o perforación múltiple con irrigación del espacio subdural), el segundo caso aquí descrito respondió adecuadamente al tratamiento médico.

La terapia antibiótica empírica debe ser acorde con el sitio primario de infección, los antecedentes del paciente y la microbiología probable. Se ha descrito un esquema triple con base en vancomicina, ceftriaxona y metronidazol para cobertura contra los principales agentes implicados. ${ }^{5}$ En ambos casos se utilizó empíricamente este esquema terapéutico y no fue posible ajuste debido a falta de aislamiento de agente causal. Sin embargo, también se han descrito otros esquemas empíricos más amplios de acuerdo con diversos factores predisponentes, como vancomicina más una cefalosporina antiseudomónica o más anfotericina B si no hay una respuesta adecuada a los 7 días de tratamiento triple. ${ }^{6}$ La duración total sugerida de la terapia con antibióticos varía de 6 a 8 semanas, como fue necesario en ambos casos. En casos específicos de infección no complicada y remoción quirúr- gica completa de un absceso bien delimitado, pueden ser suficientes cursos de 3 a 4 semanas de tratamiento. También están descritos 2 a 3 meses de antibiótico vía oral adicional para prevenir recurrencias. ${ }^{1}$

La morbilidad de las infecciones parameníngeas es alta en la mayoría de las series. La mortalidad aún es elevada en pacientes inmunodeprimidos y con retraso en el inicio del tratamiento antibiótico, ${ }^{7}$ pero los factores más importantes que influyen en la mortalidad por absceso cerebral son la edad y la condición neurológica del paciente al momento del ingreso. ${ }^{3}$

\section{CONCLUSIÓN}

El pronóstico de los pacientes con infecciones parameníngeas ha mejorado gracias al empleo de la tomografía axial computada, al perfeccionamiento de las técnicas quirúrgicas y a los avances de la antibioticoterapia. A lo largo de los años se ha observado una disminución en el número de abscesos secundarios a sinusitis $u$ otitis media y ha aumentado el número de pacientes que no ha requerido tratamiento quirúrgico. Aunque la mortalidad parece haber disminuido, un porcentaje significativo de pacientes presenta déficits neurológicos permanentes como epilepsia, déficits motor o sensitivo, defectos de campimetría, cambios en la personalidad o hidrocefalia. Por esta razón, es esencial el diagnóstico temprano y el tratamiento oportuno de este tipo de infecciones. ${ }^{1}$

\section{REFERENCIAS}

1. Feigin RD, Cherry JD, Demmler-Harrison GJ, Kaplan SL. Feigin \& Cherry's Textbook of Pediatric Infectious Diseases. 6th ed. Philadelphia: Saunders Elsevier; c2009.Chapter 38, Parameningeal infections; pp. 472-9.

2. Coria-Lorenzo JJ, Rocha-Rivera JL, Gómez-Barreto D. Absceso cerebral en los niños. Revisión. Rev Mex Ped 2002;69(6):247-51.

3. Menon S, Bharadwaj R, Chowdhary A, Kaundinya DV, Palande DA. Current epidemiology of intracranial 
González-Saldaña N y Gómez-Toscano V. Infecciones parameníngeas

abscesses: a prospective 5 year study. J MedMicrobiol 2008;1(57):1259-68.

4. Goodkin HP, Harper MB, Pomeroy SL. Intracerebral abscess in children: historical trends at Children's Hospital Boston. Pediatrics 2004;113:1765-70.

5. Gelabert-González M, Serramito-García R, García-Allut A, Cutrín-Prieto J. Management of brainabscess in children. J Paed Child Health 2008;44(12):731-5.
6. Brook I. Microbiology and management of brainabscess in children. J Ped Neurol 2004,7;2(3):125-30.

7. Gutiérrez-Cuadra M, Ballesteros MA, Vallejo A, Miñambres E, Fariñas-Álvarez C, García-Palomo JD, et al. Abscesos cerebrales en un hospital de tercer nivel: Epidemiología y factores que influyen en la mortalidad. Rev Esp Quimioter 2009;22(4):201-6. 